\title{
Apparent motion between shapes differing in location and orientation: A window technique for estimating path curvature
}

\author{
MICHAEL K. MCBEATH and ROGER N. SHEPARD \\ Stanford University, Stanford, California
}

\begin{abstract}
When a shape is alternately presented in two positions differing in both location and orientation, apparent motion tends to be experienced over a curved path. The curvature provides evidence about principles of object motion that may have been internalized in the perceptual system. This study introduces a technique for estimating deviation from a straight path. A shape was alternately presented on the two sides of a visual partition with a "window" just wide enough to accommodate the shape. Observers adjusted the location of the window to maximize the illusion of smooth passage of the shape through the window. In accordance with theoretical expectations, estimated deviations from rectilinear motion increased with the separation between the stimuli in spatial location, angular orientation, and time.
\end{abstract}

Most early studies of visual apparent motion used simple stimuli such as circular dots, which lacked welldefined spatial orientations, or horizontal rectangles, which had the same orientations. When two such stimuli were presented in alternation, observers typically reported apparent motion over a straight, connecting path. A straight path is the shortest. Moreover, to the extent that apparent motion is governed by internalized approximations of the laws of physics, a bending of the path would be expected only if observers were imposing the analogue of an external force.

Nevertheless, observers under some conditions have reported motion over a curved path. This is true both in some early studies (Brown \& Voth, 1937; Kolers, 1963; Wertheimer, 1912; see also Shipley, 1961) and, beginning with Foster (1975), in a number of more recent investigations (Anstis \& Ramachandran, 1986; Bundesen, Larsen, \& Farrell, 1983; Farrell, 1983; Mori, 1982; Proffitt, Gilden, Kaiser, \& Whelan, 1988; Shepard \& Zare, 1983). A condition often found to induce curved apparent motion is that in which the two alternately presented shapes (such as rectangles) differ in orientation as well as in location (see Foster, 1975).

A curved path under this condition is consistent with the notion that principles of geometry have dominated those of physics in the evolutionary internalization of external constraints (Shepard, 1984). For any two positions of an asymmetric shape in a plane, there is a unique fixed point around which the shape can be rigidly rotated back and forth between the two positions. This is known as

This research was supported by NSF Research Grant BNS 85-11685 to the second author. We thank Dennis Proffitt for suggestions concerning the manuscript. Correspondence concerning this article should be addressed to either author, Department of Psychology, Building 420, Stanford University, Stanford, CA 94305-2130.
Euler's theorem-the two-dimensional special case of Chasles's theorem for three-dimensional space (see Carlton \& Shepard, 1989a; Shepard, 1984). In this twodimensional case, the two presented positions of a shape determine a unique connecting circular path in the plane. Thus, the empirical estimation of the deviation from rectilinear apparent motion has an important bearing on theoretical issues concerning which principles of motion have been internalized in the perceptual system.

The methods of estimating deviation from rectilinearity have so far been rather limited. Primarily, observers have either simply reported whether the motion between the two alternately presented shapes appeared to follow a curved path, or reported whether the apparently moving shape seemed to pass through the particular position of a third, probe shape momentarily presented between them (Foster, 1975; Mori, 1982; Proffitt et al., 1988; see also Robins \& Shepard, 1977). The reports obtained by the first of these methods are merely qualitative, and the paths determined by the second method, though quantitative, can be affected by the presentation of the intermediate probe-a third stimulus potentially contributing to the path of the apparent motion itself (Anstis \& Ramachandran, 1986, 1987; Burt \& Sperling, 1981). For example, the insertion of the third stimulus may effectively halve the separations between stimuli in time, distance, and orientation-variables that have all been found to influence the experienced path of apparent motion (Farrell, 1983; Foster, 1975; Mori, 1982; Proffitt et al., 1988).

In this paper, we introduce a "window" technique for obtaining quantitative estimates of the deviation of apparent motion from rectilinearity. Instead of adjusting the location and orientation of an intermediate flashing replica of the apparent-motion stimulus, observers adjust the location of a window in a visual partition permanently separating the shapes. The perceptually optimal path is in- 
ferred from the placement of the window that yields the most compeling appearance of smooth motion through it. This method does not rule out the possibility that the method of measurement affects the to-be-measured curvature. For example, the placement of the wall halfway between the two stimuli could conceivably increase the salience of the circular path because the wall passes through the center of the circle. Still, an enduring window seems a less powerful stimulus for influencing the path of motion than does a third, flashing replica of the apparent-motion stimulus itself. Moreover, a window, unlike a replica flashed in a particular orientation, does not force any intermediate orientation on the moving shape. (Although we do not attempt a direct comparison between the window and probe techniques here, we do show that the window technique offers a reliable alternative method for determining the departure of trajectory from a straight path.)

We also report preliminary evidence concerning possible temporal determinants of the path of apparent motion. The minimum stimulus onset asynchrony (SOA; or minimum interstimulus interval, ISI, if the stimulus duration is fixed) yielding good apparent motion generally increases with the extent of the motion (Kolers, 1963; Korte, 1915; Neuhaus, 1930). Presumably, this is because more time is needed for the impletion of a longer path. Indeed, the increase tends to be linear, whether the motion itself is rectilinear (Corbin, 1942; see Shepard, 1984, p. 427) or rotational (Bundesen et al., 1983; Farrell, 1983; Farrell, Larsen, \& Bundesen, 1982; Farrell \& Shepard, 1981; Shepard \& Judd, 1976). Moreover, Shepard and Zare (1983), using a new technique of "pathguided" apparent motion, demonstrated that even when the straight distance between two alternately presented dots was fixed, the critical SOA for apparent motion over an experimenter-determined curved path increased linearly with the length of that curved path. In this paper, we investigate whether, conversely, lengthening ISI (and, hence SOA for a fixed stimulus duration) increases curvature and, hence, length of experienced path of motion.

\section{METHOD}

\section{Stimuli and Procedure}

For this first investigation of the window technique, we used an IBM XT computer to display the stimuli. Despite the limited speed and resolution of the graphic display capabilities of this computer, we obtained results that were sufficiently encouraging to be reported here. Moreover, these results are consistent with results that one of us (M.K.M.) is currently obtaining using the window technique with a more powerful display system.

For this initial exploration, we selected three quite different shapes for apparent-motion stimuli (Figure 1a). These include the relatively narow line-drawn shape previously used by Farrell (1983), a somewhat less narrow silhouette shape resembling the irregular polygons used by Farrell and Shepard (1981), and a wider "Pac Man" shape that approximated, within the resolution of our display system, a circle with a pie-shaped segment removed (in order that the two stimuli could have distinctly different orientations and, hence, could induce a curved path of motion). The narrower shapes were always oriented so that their axes of elongation passed through the center of rotation uniquely specified for the presented pair by kinematic geometry (an arrangement that may favor extraction of

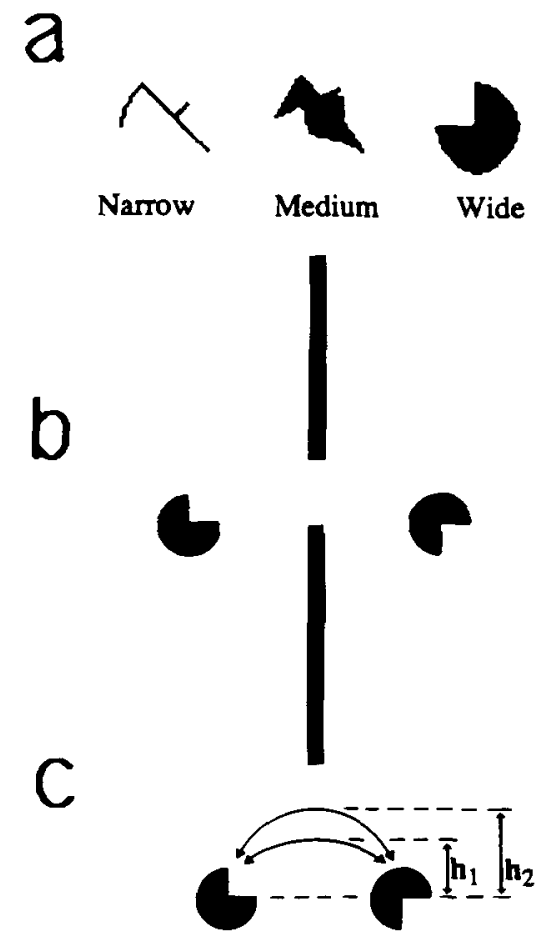

Figure 1. Stimuli used in the present experiment. (a) The three shapes differing in width or elongation. (b) Layout of a visual display, showing two alternately presented positions of a shape to the left and right of a vertical partition. Observers adjusted the height of the window until the shape seemed to pass most smoothly through it. (c) Schematic portrayal of curved paths of differing beights, $h_{1}$ and $h_{\mathbf{2}}$.

that fixed point by the visual system; see Proffitt et al., 1988). The "Pac Man" shape was always oriented so that it was not symmetric about a vertical axis, thus reducing the likelihood of apparent rotational motion in depth (cf. Kolers \& Green, 1984; Kolers \& Pomerantz, 1971; Shepard \& Judd, 1976). At a viewing distance of approximately $80 \mathrm{~cm}$, the maximum diameter of each shape subtended about $1.5^{\circ}$ of visual angle.

On each trial, one of these three shapes was alternately presented, in two different orientations, to the left and right of a vertical partition. A gap or window in the partition was just large enough to admit the rigid passage of the shape (Figure $1 b$ ). By means of a joystick, the observer adjusted the vertical height of the window to obtain the strongest illusion of smooth motion of the object back and forth through the window. Our measure of deviation from rectilinear apparent motion was height (in degrees of visual angle) of the center of the adjusted window above the straight line that would connect the centers of the two alternately displayed stimuli (see Figure 1c).

Each of the two alternately displayed stimuli remained on for a fixed duration of $550 \mathrm{msec}$. On different trials, however, we imposed different ISIs, ranging from 0 to $200 \mathrm{msec}$ (in 50-msec steps), which yielded SOAs ranging from 550 to $750 \mathrm{msec}$. We also included three equally spaced separations between the centers of the two alternately presented shapes, subtending (at the approximately $80-\mathrm{cm}$ viewing distance) about $3^{\circ}, 6^{\circ}$, and $9^{\circ}$ of visual angle. Finally, we included both $90^{\circ}$ and $180^{\circ}$ angular differences in the orientations of the two alternately presented shapes. According to kinematic geometry, stimuli differing by $180^{\circ}$ yield an ambiguity in which the motion can curve either above or below the straight connecting line. We primed the observers for the upwardly curved interpretation throughout the experiment by (1) placing the two 
stimuli closer to the bottom of the display screen, (2) explicitly requesting the subjects to try for a good placement of the window either at or above a straight line between the two presented positions of the shape, and (3) choosing the half of the trials with $90^{\circ}$ stimuli so that the curvature predicted by kinematic geometry would be upward (as illustrated in Figure 1c; see also Carlton \& Shepard, 1989a).

To accommodate cases in which the observer could not experience a well-defined path of motion or could not suppress alternative paths of motion, we had observers rate their confidence in their final placement of the window on each trial on a 3-point scale. A rating of 3 represented high confidence and a well-defined path, a rating of 1 represented low confidence and a poorly defined path, and a rating of 2 indicated intermediate confidence and definition of path.

We presented each combination of levels of the independent variables twice to each observer, in random order, yielding a total of 3 shapes $\times 3$ spatial separations $\times 2$ angular differences $\times 5$ ISIs $\times 2$ replications, for a total of 180 trials per observer. The experiment required an average of about 45 min per observer.

\section{Subjects}

Ten 18- to 22-year-old undergraduate Stanford students enrolled in an introductory psychology course served as observers. Six were male and 4 were female. All 10 subjects had normal or correctedto-normal vision, and were not informed of our notions about curvature of the path of motion.

\section{RESULTS}

\section{Curvature of Experienced Path}

The observers set the height of the adjustable window well above a straight line between the centers of the two alternately presented stimuli (i.e., as illustrated in Figure 1c). Indeed, the magnitude of this upward displacement of the window averaged between one fourth and one third of the distance between the centers of the alternately presented shapes.

According to a multiple regression analysis used to assess the relative contribution to path curvature of the various independent variables, the overall deviation from a rectilinear path was statistically reliable, and remained so whether the analysis included all trials $[F(4,1795)=385$, $p<.001]$ or only those trials for which the observers gave the highest confidence rating (3) for their window placement $[F(4,985)=313, p<.001]$.

The obtained deviation was, however, generally less than that prescribed by Euler's theorem of kinematic geometry-averaging between $60 \%$ and $75 \%$ of the predicted values when the alternately presented shapes differed widely in angular orientation (i.e., by $180^{\circ}$ ) and in spatial location (i.e., by $6^{\circ}$ of visual angle or more). In the Discussion section, we consider possible reasons why the deviations from a straight path, though appreciable and reliable, fell short of those prescribed by kinematic geometry.

\section{Dependence of Curvature on the Independent Variables}

Figure 2 summarizes how the estimated departures from a straight path depended on the principal independent variables-namely, spatial separation between the alter-
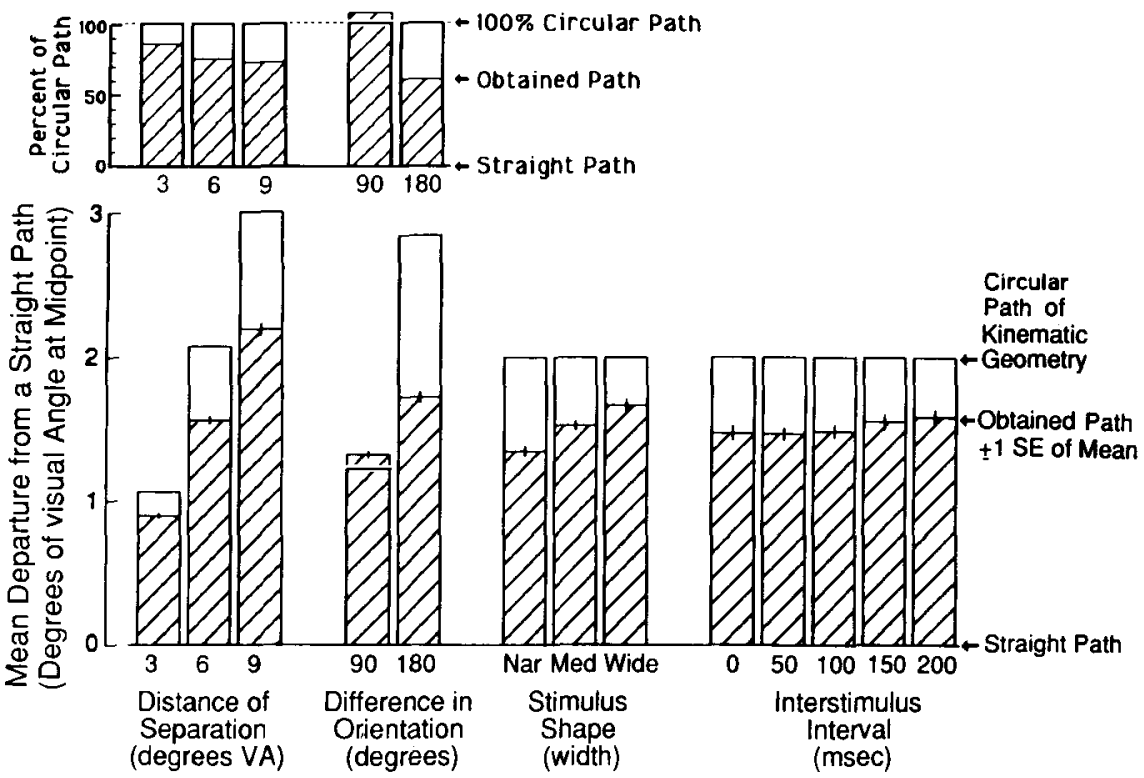

Figure 2. Mean departures of the center of the adjusted window above a straight path between the centers of the two alternately presented stimuli. Each bar shows the obtained departure for one level of one of the four independent variables. The departure is averaged over all trials except those on which observers gave the lowest confidence rating (i.e., "1"). The total height of each bar indicates the departure prescribed by kinematic geometry. The height of the hatched portion indicates the obtained departure estimated from the observers' adjustments of the window. The short vertical line at the top of the shaded portion indicates \pm 1 standard error of that obtained mean. In the upper left, the same data are replotted, for the tested separations and angular differences, as a percentage of the heights of the circular paths predicted by kinematic geometry. 
nately presented stimuli, angular difference in their orientations, degree to which the stimuli were wide (rather than narrow or elongated), and temporal separation (ISI). The total heights of the vertical bars above the baseline represent the departures corresponding to the circular paths prescribed by kinematic geometry. The heights of the hatched portions of the bars represent the departures inferred from the observers' mean settings of window height. The small line segment at the top of each shaded portion indicates $\pm 1 S E M$ of these settings.

The multiple regression analysis revealed a reliable dependence of the height of the curved path on each of the independent variables. For the $55 \%$ of the trials on which the observers gave the highest confidence rating of $3\left(R^{2}\right.$ $=.560 ; d f=985)$ and curvature significantly increased with spatial separation $(t=33.5, p<.001)$, angular difference $(t=9.63, p<.001)$, width of shape $(t=$ $7.51, p<.001)$, and ISI $(t=2.32, p<.05)$. These dependencies were also evident, though somewhat weakened, when trials with the lower confidence ratings of 2 and 1 were successively included $\left(R^{2}=.489\right.$ and $.462 ; d f=1629$ and 1795 , respectively). All 10 observers exhibited a similar pattern of results, but there were significant individual differences in the extent of path curvature $[F(9,980)=11.49, p<.001]$, with $R^{2}$ ranging from $.36(d f=45)$ to $.77(d f=113)$, for individual observers on high-confidence trials.

Figure 2 shows that the paths estimated for all values of the independent variables were closer to the circular paths predicted by kinematic geometry than to rectilinear paths. As we have mentioned, however, the estimated paths fell increasingly short of the predicted curvatures as the distances or angular disparities between the stimuli increased. This is graphically illustrated in the upper left of Figure 2, where the obtained data are replotted as percentages of the predicted departures from a straight path.

As Figure 2 shows, the effect of ISI on curvature of path was extremely small. Nevertheless, the trend toward increasing deviation from a straight motion with increasing ISI, though not statistically significant when trials with all three confidence ratings were included $[t(1795)=$ 1.22 ], became significant when the $90 \%$ of trials having the two higher ratings of confidence ( 3 and 2 ) were analyzed separately $[t(1613)=2.87, p<.01]$ and, as already noted, when only the $55 \%$ of trials having the highest rating of confidence (3) were analyzed $[t(985)=$ $2.32, p<.05]$. Moreover, the relationship between ISI and curvature was in the direction expected on certain theoretical grounds (considered in the ensuing discussion) for 8 of the 10 subjects, with the results for 4 of those subjects attaining $p<.05$.

\section{DISCUSSION}

We take the results-obtained by our new, window method of estimating path curvature-to provide new evidence that apparent motion generally departs from a straight line when two alternately presented shapes differ in orientation as well as in location. Such a departure is to be expected if apparent motion is governed by internalized principles of kinematic geometry. The circular motion prescribed by Euler's theorem departs further from a straight line as either the linear separation or the angular difference between the stimuli increases (Carlton \& Shepard, 1989a).

This evidence for path curvature cannot plausibly be explained as arising, instead, from a default tendency of observers to position the window toward the middle of its adjustable range - a range in which only the lowest extreme corresponds to a straight path. Such a default tendency, if operative, should have manifested itself most strongly when the observers gave their lowest ratings of confidence about window placement. Our results were just the opposite. When the observers gave lower confidence ratings, they also produced reliably lower window placements, corresponding to straighter paths of motion. As further evidence against such a default tendency, when the observers gave high confidence ratings, the heights of their window placements above a straight path increased systematically with each of the independent variables of spatial separation, angular difference, and ISI. Moreover, as we shall explain, these are just the increases that we expected on theoretical grounds.

Stimulus shape (which we have very provisionally referred to as "width" here) was varied only in order to achieve some degree of generality and not to test any firm theoretical predictions about the effect of shape on the path of apparent motion. Nevertheless, both empirical results (Proffitt et al., 1988; see also McBeath, 1989) and theoretical considerations (Carlton \& Shepard, 1989b) have subsequently indicated that the relative orientations of the salient axes of an object, whether axes of elongation or of symmetry, affect the path of motion.

The very small, but statistically reliable, increase in departure from a straight path with increasing ISI, also, is not deducible from kinematic geometry alone. Kinematic geometry selects certain paths as simplest regardless of temporal considerations and, hence, places no constraints on speed or duration of motion over such paths. A dependence of path curvature on ISI might, however, be explainable as a manifestation of the central Helmholtzian principle that perceptual experience is the result of the simultaneous optimization of consistency with (1) what is likely in the external world and (2) the evidence immediately available at the sensory surfaces (Helmholtz, 1857/1962). For a given ISI, a longer path would entail more rapid motion over that path and, hence, less time for stimulation of each local receptor along the way. The absence of excitation of intermediate receptors during an appreciable ISI is therefore more consistent with external motion over a longer and, hence, more curved path.

Korte's (1915) third law of apparent motion might also partially account for the increase in path curvature with increase in ISI. According to the relevant extension of this law (e.g., see Attneave \& Block, 1973; Shepard, 
1984; Shepard \& Cooper, 1982), SOA (and, hence, ISI if the stimulus durations are fixed) increases linearly with length of the path, which may be variously curved (Farrell, 1983; Shepard \& Zare, 1983). (Korte's law may be a consequence of limitations on the velocity of neural processing and propagation, which may limit the length of a path that can be traversed in a given time; see Shepard, 1981, 1984, 1989). A display for which kinematic geometry prescribes a more curved, longer path of transformation should therefore require a longer SOA and hence (for a fixed stimulus duration) a longer ISI. If SOA is too short for completion of the circular path, the perceptual system may connect the alternately displayed stimuli through a path that is flattened and hence shortened enough to allow motion to be completed within the time available (as suggested, also, by the results of Farrell et al., 1982; Proffitt et al., 1988).

The same consideration may explain the general tendency of our estimated curvatures to fall increasingly short of the predictions of kinematic geometry as the distances or angular differences between the alternately presented stimuli increased. The averaged estimates of height would be reduced to the extent that they include trials in which only a flatter, and hence shorter, path could be completed within the available time. Quite generally, we would expect a given visual display to engage internalized principles of kinematic geometry only when the spatiotemporal conditions of the display fall within the limits required for the neural integration and processing of visual information (see Shepard, 1984). Measured performance may fail to reveal the competence embodied in perceptually internalized principles of kinematic geometry to the extent that the alternately presented stimuli become too widely separated in location, orientation, or time (ISI), or to the extent that the temporal interval available for the establishment of a correspondence between the two stimuli (SOA) becomes too brief.

\section{REFERENCES}

Anstis, S., Ramachandran, V. S. (1986). Entrained path deflection in apparent motion. Vision Research, 26, 1731-1739.

ANSTIS, S., \& RAMACHANDRAN, V. S. (1987). Visual inertia in apparent motion. Vision Research, 27, 755-764.

ATtNeave, F., Block, G. (1973). Apparent motion in tridimensional space. Perception \& Psychophysics, 13, 301-307.

Brown, J. F., Voth, A. C. (1937). The path of seen movement as a function of the vector-field. American Journal of Psychology, 49, 543-563.

Bundesen, C., Larsen, A., Farrell, J. E. (1983). Visual apparent movement: Transformations of size and orientation. Perception, 12, 549-558.

Burt, P., Sperling, G. (1981). Time, distance, and feature tradeoffs in visual apparent motion. Psychological Review, 82, 171-195.

Carlton, E. H., \& ShePard, R. N. (1989a). Psychologically simple motions as geodesic paths: 1 . Asymmetric objects. Manuscript submitted for publication.

Carlton, E. H., ShePard, R. N. (1989b). Psychologically simple motions as geodesic paths: 2. Symmetric objects. Manuscript submitted for publication.

Corbin, H. H. (1942). The perception of grouping and apparent movement in visual depth. Archives of Psychology, 273, 1-50.

FARRELL, J. E. (1983). Visual transformations underlying apparent movement. Perception \& Psychophysics, 33, 85-92.

Farrell, J. E., LARSen, A., Bundesen, C. (1982). Velocity constraints on apparent rotational movement. Perception, 11, 541-546.

FARRELl, J. E., \& ShePARD, R. N. (1981). Shape, orientation, and apparent rotational motion. Jourmal of Experimental Psychology: Human Perception \& Performance, 7, 477-486.

FosTER, D. H. (1975). Visual apparent motion and some preferred paths in the rotation group SO(3). Biological Cybernetics, 18, 81-85.

HeLmholTZ, H. voN. (1962). Treatise on physiological optics (Vol. 3). New York: Dover. (Original work published 1857)

Kolers, P. A. (1963). Some differences between real and apparent visual movement. Vision Research, 3, 191-206.

holers, P. A., \& GreEN, M. (1984). Color logic of apparent motion. Perception, 13, 417-428.

Kolers, P. A., \& Pomerantz, J. R. (1971). Figural change in apparent motion. Joumal of Experimental Psychology, 87, 99-108.

KORTE, A. (1915). Kinematoskopische Untersuchungen. Zeitschrift für Psychologie, 72, 194-296.

MCBEATH, M. K. (1989). The effect of object shape on the path of apparent motion. Manuscript in preparation.

MORI, T : (1982). Apparent motion path composed of a serial concatenation of translations and rotations. Biological Cybernetics, 44, 31-104.

Neuhaus, W. (1930). Experimentelle Untersuchung der Scheinbewegung. Archiv fur die gesamte Psychologie, 75, 315-458.

Proffitt, D. R., Gilden, D. L., Kaiser, M. K., Whelan, S. W. (1988). The effect of configural orientation on perceived trajectory in apparent motion. Perception \& Psychophysics, 43, 465-474.

RoBins, C., \&HEPARD, R. N. (1977). Spatio-temporal probing of apparent rotational movement. Perception \& Psychophysics, 22, 12-18.

ShePARD, R. N. (1981). Psychophysical complementarity. In M. Kubovy \& J. Pomerantz (Eds.), Perceptual organization (pp. 279-341). Hillsdale, NI: Erlbaum.

SHEPARD, R. N. (1984). Ecological constraints on internal representation: Resonant kinematics of perceiving, imagining, thinking, and dreaming. Psychological Review, 194, 417-447.

SHEPARD, R. N. (1989). Internal representation of universal regularities: A challenge for connectionism. In L. Nadel, L. A. Cooper, P. Culicover, \& R. M. Harnish (Eds.), Neural connections, mental computation (pp. 104-134). Cambridge, MA: MIT Press/Bradford Books.

SHEPARD, R. N., \& CoOPER, L. A.(1982). Mental images and their transformations. Cambridge, MA: MIT Press/Bradford Books.

SHEPARD, R. N., JudD, S. A. (1976). Perceptual illusion of rotation of three-dimensional objects. Science, 191, 952-954.

ShePARD, R. N., ZARe, S. L. (1983). Path-guided apparent motion. Science, 220, 632-634.

SHIPLEY, T. (1961). Classics in psychology. New York: Philosophical Library.

WERTHEIMER, M. (1912). Experimentelle Studien über das Sehen von Bewegung. Zeitschrift fur Psychologie, 61, 161-265.

(Manuscript received October 24, 1988; revision accepted for publication March 23, 1989.) 